\title{
Multiple sclerosing hemangioma of the right lung in a 23-year-old female patient: A case report and review of the literature
}

\author{
HAN LUO $^{1 *}$, LIJIE MA ${ }^{1 *}$, YIHUA CHEN ${ }^{2}$, TINGXIU ZHANG ${ }^{1}$, DAQING PENG ${ }^{1}$, \\ ZHIWEI LI ${ }^{1}$, XIAOPING DAN ${ }^{1}$, ZHENLIANG XIAO $^{1}$ and ZHANG CHEN ${ }^{1}$ \\ Departments of ${ }^{1}$ Pulmonary and Critical Care Medicine, and ${ }^{2}$ Pathology, General Hospital of \\ Western Theater Command, Chengdu, Sichuan 610083, P.R. China
}

Received August 7, 2018; Accepted June 27, 2019

DOI: $10.3892 / \mathrm{mco} .2020 .1986$

\begin{abstract}
Pulmonary sclerosing hemangioma (PSH) is a relatively uncommon benign tumor of the lung, predominantly affecting young and middle-aged women. In the majority of the patients, PSH is incidentally found on physical examination and typically presents as a solitary nodule with smooth borders, as it is generally asymptomatic or lacks typical symptoms. In the present case, a 23 -year-old woman was incidentally diagnosed with pulmonary nodules during routine physical examination and reported suffering from intermittent fevers for $>2$ months. The patient received antituberculosis therapy for 1 year; however, a computed tomography imaging examination revealed that the lesions had progressed. Finally, the patient underwent thoracoscopic lung biopsy followed by histopathological examination and the lesions were diagnosed as multiple sclerosing hemangioma. The aim of the present study was to review the relevant literature in order to improve our understanding of PSH.
\end{abstract}

\section{Introduction}

Pulmonary sclerosing hemangioma (PSH) is an uncommon benign tumor of the lungs, first reported by Liebow and Hubbell in 1956 (1). Its origin has been suggested to be vascular, mesothelial, mesenchymal, epithelial and neuroendocrine (1-5), but immunohistochemical examination suggests that PSH is derived from primitive respiratory epithelium. It primarily affects Asian women and the female: Male ratio is 5:1 (6,7). PSH predominantly presents as a solitary, sharply

Correspondence to: Dr Zhang Chen or Professor Zhenliang Xiao, Department of Pulmonary and Critical Care Medicine, General Hospital of Western Theater Command, 270 Tianhui Road, Rongdu Avenue, Jinniu, Chengdu, Sichuan 610083, P.R. China

E-mail: chenzhang10260@163.com

E-mail: xiaozhenliang2001@aliyun.com

*Contributed equally

Key words: pulmonary sclerosing hemangioma defined slow-growing mass, although it may present as multiple lesions (8). On imaging, PSH appears as a mass with distinct margins, and the majority of the patients are asymptomatic. Definitive diagnosis requires resection and postoperative histopathological examination. Due to its atypical image presentations, PSH may be easily misdiagnosed as a malignant tumor prior to surgery, with a misdiagnosis rate that ranges from 25 to $56 \%$ (8). We herein report a case of PSH and perform a review of the literature to explore the clinical management of PSH.

\section{Case report}

A 23-year-old unmarried woman was hospitalized after a mass was incidentally found in her right lung during routine physical examination. The patient had a history of allergic reaction to penicillin and cephalosporin. The patient was in good general health and had no unhealthy habits, such as drug or alcohol abuse or smoking. The patient's personal, menstrual and family history were unremarkable.

The chest computed tomography (CT) of the patient revealed several shadows in the right lung during a physical examination in October 2014. The patient visited Qingdao Chest Hospital (Qingdao, China) for further evaluation and the $\gamma$-interferon release testing was found to be positive. The patient was diagnosed with tuberculosis and was started on antituberculosis treatment with rifampicin, armazide, ethambutol and pyrazinamide. The patient started to experience intermittent fevers over the next 2 months of antituberculosis treatment, with the highest temperature reaching $39^{\circ} \mathrm{C}$, usually improving at night and relieved by ibuprofen. Interestingly, the patient had no history of cough, expectoration, dyspnea, chest pain, palpitations or weight loss. The patient underwent regular chest $\mathrm{CT}$ re-examinations during the antituberculosis treatment, which revealed no changes in the lesions. The patient accepted CT examination again in March 2015 and no obvious changes were evident in the images of the right lung. Percutaneous lung biopsy was performed, and histopathological examination revealed inflammatory and hyperplastic changes. Most importantly, the antituberculosis treatment was continued based on the results of postoperative pathology.

The patient first visited Chengdu Military General Hospital (Chengdu, China) at the end of September 2015, and a CT 

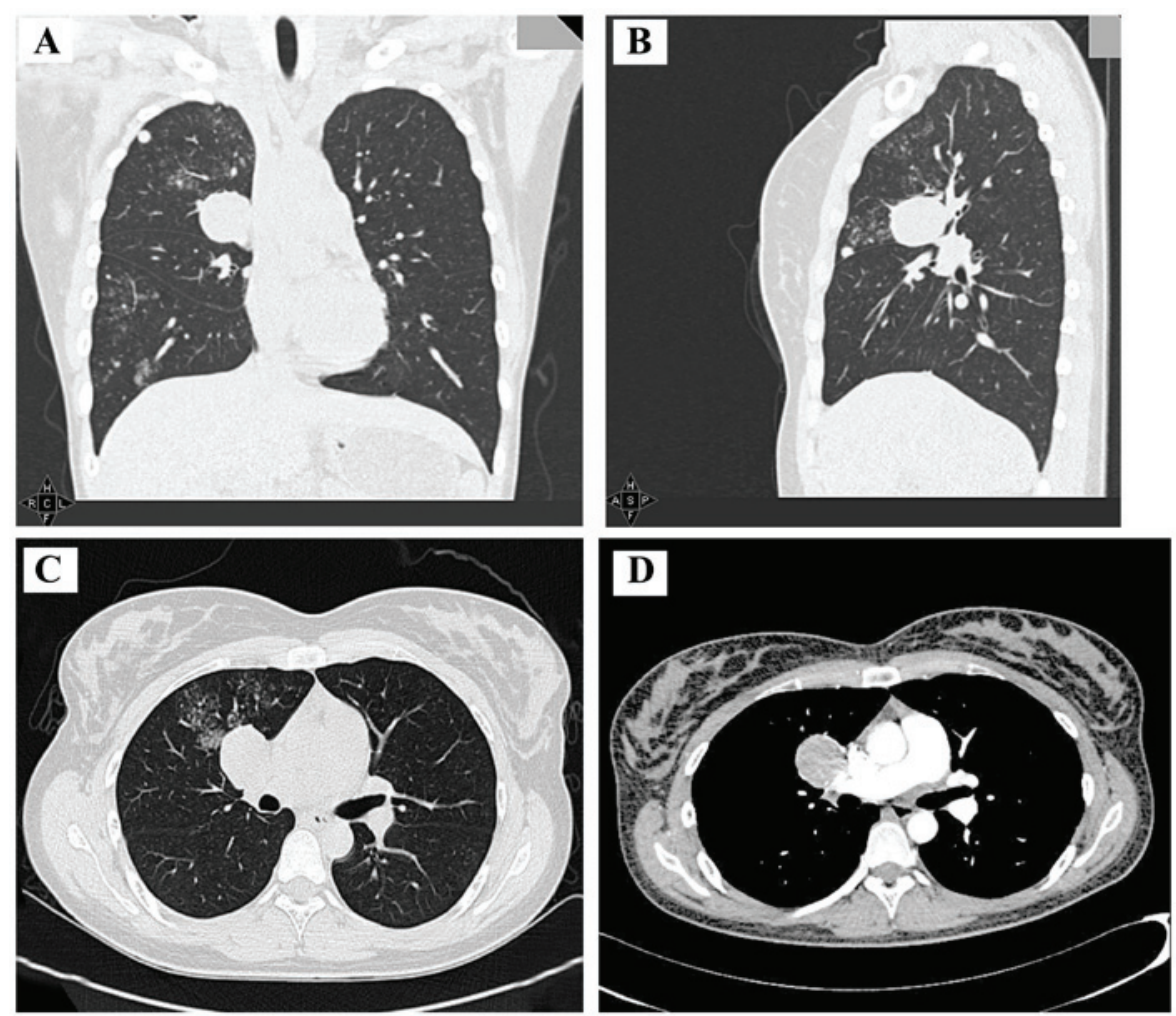

Figure 1. CT examination performed in August 2017. Enhanced CT examination revealed nodular slices in the pulmonary hilum surrounded by exudative changes and satellite lesions. Uneven enhancement and the vascular border sign were also observed. (A) coronal view, (B) sagittal view, (C) transverse lung window and (D) transverse mediastinal window. CT, computed tomography.

scan revealed a circular mass of soft tissue density in the right upper pulmonary hilum. In addition, the right lung exhibited scattered dot films and small nodules, consistent with the imaging findings of pulmonary tuberculosis. Admission was recommended for further evaluation, but the patient declined due to work responsibilities. Antituberculosis treatment was continued on an outpatient basis and further chest CT scans were performed in June 2016, April 2017 and August 2017. The CT scan performed in August 2017 revealed an increased number of dot films and small nodules in the right lung, with the additional appearance of flakes of blurry shadows (Figs. 1 and 2). The patient was then admitted to the hospital and further examinations were undertaken.

The physical examination of the patient was normal. The findings on routine blood and urine tests and bronchoscopy were normal, apart from the results of the $\gamma$-interferon release testing. In order to reach a definitive diagnosis, positron emission tomography (PET)-CT was performed (Fig. 3), revealing the presence of multiple nodules of varying sizes and densities. Furthermore, increased fluorodeoxyglucose uptake was observed in some of the nodules with calcification. As tuberculosis recurrence was first suspected, the patient underwent thoracoscopic surgery with right lung nodulectomy. Based on the postoperative pathological examination of the nodules, the lesions were diagnosed as multiple PSH, and four different histological patterns were identified: Papillary, solid, sclerotic and hemorrhagic (Fig. 4A). Immunohistochemical evaluation of the lesions revealed positive staining for thyroid transcription factor (TTF)1 (Fig. 4B) and cytokeratin (CK) 8/18 (Fig. 4C), but negative staining for smooth muscle actin (Fig. 4E). The Ki-67 labeling index was 1\%. (Fig. 4D).

All examinations and medical procedures were approved by the patient and her family.

\section{Discussion}

PSH is a benign tumor of the lungs with variable histological characteristics. PSH was originally considered to be of vascular origin due to the obvious characteristics of hemangioma. After the first description in 1956 by Liebow and Hubbel (1), several theories regarding its origin were introduced, including vascular (1), mesothelial (2), mesenchymal (3), epithelial (4) and neuroendocrine (5). Currently, the results of the immunohistochemical examination suggest that PSH is derived from primitive respiratory epithelium. $\mathrm{PSH}$ has been reported to be more prevalent in East Asian rather than in Western countries, and typically affects middle-aged adults and exhibits a female predominance, with women comprising $\sim 80 \%$ of PSH patients $(6,7)$. In the majority of the cases $(\sim 95 \%)$, PSH presents as a solitary slow-growing mass located in the peripheral lung parenchyma, except for $4 \%$ of the cases that present as multiple lesions (8). The tumor size may vary from 0.3 to $8 \mathrm{~cm}$ in greatest diameter, but the majority measure $<3 \mathrm{~cm}$. PSH is almost always benign, with only $4 \%$ of PSHs invading the visceral pleura and only $1 \%$ growing into the mediastinum or the bronchial cavity. The right lung, particularly the lower lobe, has been reported to be most commonly affected by PSH. The findings on CT scans include a mass with distinct margins and increased density on contrast-enhanced CT. The majority 

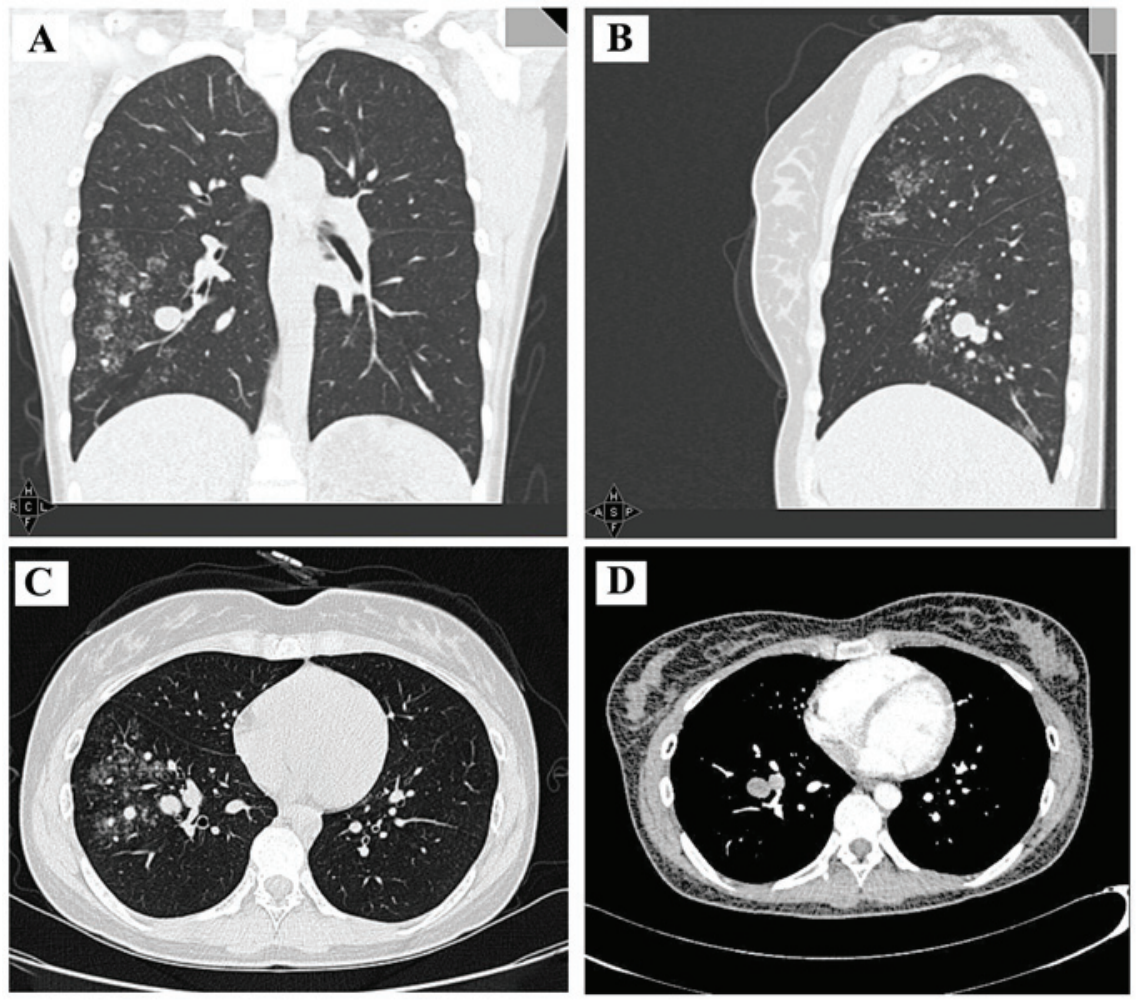

Figure 2. Computed tomography examination performed in August 2017. Several nodules were found in the peripheral zone of the right lower lung that were surrounded by exudative changes and satellite lesions. (A) coronal view, (B) sagittal view, (C) transverse lung window and (D) transverse mediastinal window.
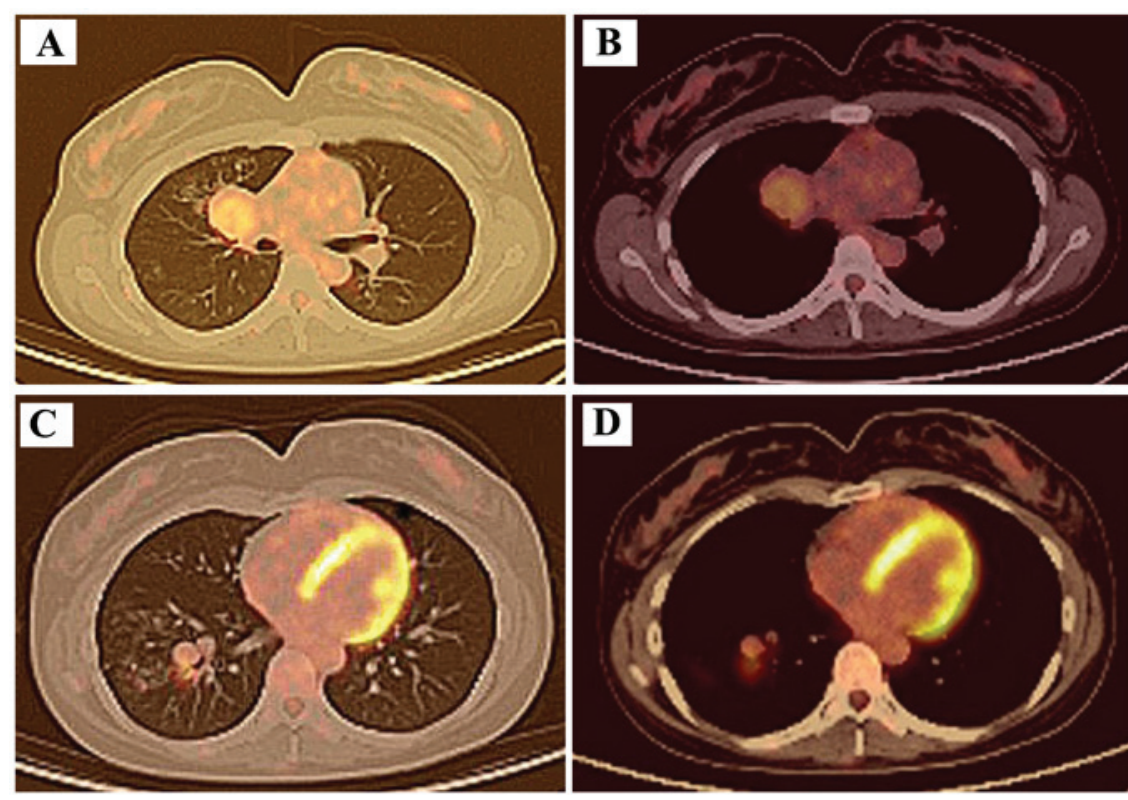

Figure 3. Positron emission tomography-computed tomography examination performed in August 2017. (A) PET-CT cross-sectional lung window indicated nodules and shadows in the right lung with increased fluorodeoxyglucose metabolism. (B) PET-CT cross-sectional mediastinal window indicated nodules and shadows associated with increased metabolism of fluorodeoxyglucose in the right lung. (C) PET-CT cross-sectional lung window indicated multiple nodules in the right lower lung with increased metabolism of fluorodeoxyglucose. (D) PET-CT cross-sectional mediastinal window indicated multiple nodules in the right lower lung with increased fluorodeoxyglucose metabolism.

(80\%) of PSHs are asymptomatic or present with cough, hemoptysis and, occasionally, chest pain.

Histologically, PSH includes two essential types of tumor cells: Round stromal cells and surface cuboidal cells. Round stromal cells with absent or rare clear nucleoli are small and well-circumscribed. However, the surface cuboidal cells lining papillary structures exhibit characteristics of bronchiolar epithelium and type II pneumocytes. These cells may display different degrees of nuclear heterogeneity. Therefore, they may be arranged into four different histological patterns: Papillary, 

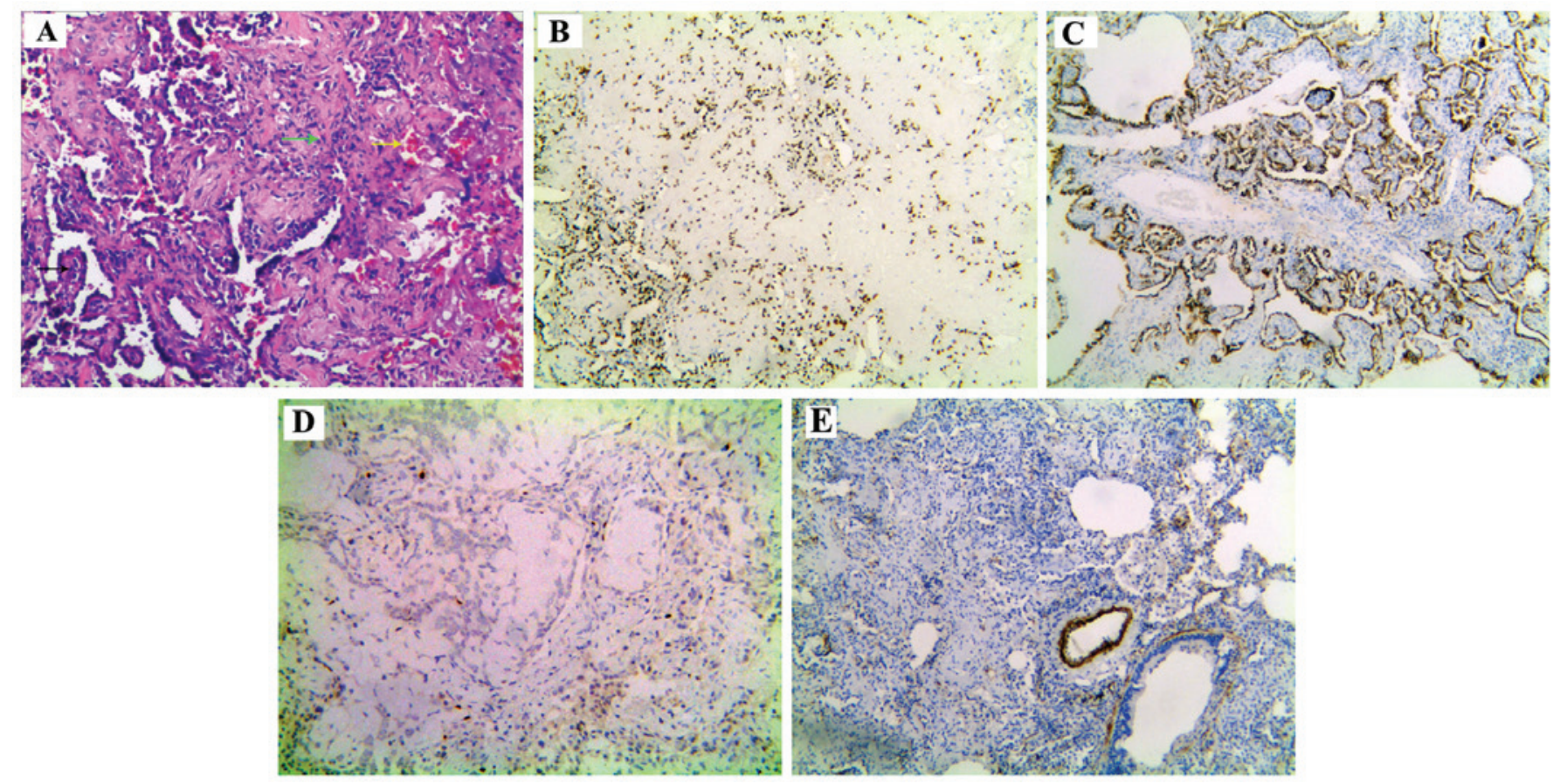

Figure 4. Pathological examination results. (A) Four different histological patterns were observed on hematoxylin and eosin staining: Papillary structures (black arrow), hyaline denatured collagen (white arrow), solid structures (green arrow) and a hemorrhagic focus of hemosiderin pigment (yellow arrow). Immunohistochemical examination of the lesions revealed positive staining for (B) thyroid transcription factor 1 and (C) cytokeratin 8/18. (D) The Ki-67 labeling index was $1 \%$. (E) Staining for smooth muscle actin was negative.

solid, sclerotic and hemorrhagic. In the present case, although the immunohistochemical examination revealed that the two cell types were positive for TTF-1 and epithelial membrane antigen, the expression of pancytokeratin $\mathrm{A}$ and various $\mathrm{CKs}$ were different in the two cell types, as pancytokeratin A and CK staining was only observed in the surface cells of the mass. Molecular studies confirmed that both cell types were clonal in nature, demonstrating that PSH is a true neoplasm.

The origin and progression of PSH remain controversial. The current consensus is that primary papillary alveolar epithelial cells proliferate and cover the fibrous tissue of the alveolar wall. At this stage, the epithelial cells may be atypical, but not malignant. Subsequently, sclerosis of interstitial alveolar cells is aggravated, leading to implantation of capillaries. As a consequence, hemorrhage occurs in the residual alveolar cavity, causing a hemosiderin reaction or accumulation of vacuolar macrophages and alveolar destruction.

Our patient had no obvious causes or positive symptoms in the early stages of the disease. Several shadows were located in the right pulmonary hilum, and the right lower lung was surrounded by inflammatory exudations that were consistent with the critical changes of pulmonary tuberculosis. Antituberculosis therapy was recommended by the first attending physician, as well as by other consultants thereafter, based on the consideration that China is a region of high incidence of tuberculosis. The patient was positive for whole-blood $\gamma$-interferon release assay. After ruling out malignant tumors, common bacteria in the region, and infection by atypical pathogens, tuberculosis remained the most likely diagnosis. The patient also developed high fevers in the afternoon, which was consistent with the typical symptoms of tuberculosis. More importantly, the high fever disappeared after antituberculosis treatment, suggesting treatment efficacy. However, the pulmonary lesions increased after 6 months of treatment and the biopsy results indicated inflammatory changes in the lungs. Antituberculosis therapy was continued, as the patient reported no feeling of discomfort and the duration of antituberculosis therapy was deemed insufficient. Unfortunately, the CT scan performed in August 2017 revealed that the pulmonary lesions had increased after the patient completed a 1-year course of antituberculosis treatment. In addition, the PET-CT results indicated recrudescence of tuberculosis. The clinical manifestations of the patient were carefully analyzed and it was concluded that the diagnosis of tuberculosis could not fully explain the patient's condition and all the imaging findings. Thoracoscopic surgery and right lung nodulectomy were performed in order to pathologically diagnose the patient. Surgical excision is considered the best choice for single PSH, while long-term follow-up is required to determine the prognosis of multiple PSHs.

In conclusion, we herein present a rare case of PSH with symptoms of discontinuous fever and multiple pulmonary nodules that was misdiagnosed as tuberculosis in its initial stages. Following adequate antituberculosis therapy, the lesions progressed but the patient remained asymptomatic. Although bronchoscopy and lung biopsy through percutaneous paracentesis were performed, there were no positive results. Finally, thoracoscopy and immunohistochemical examination were used to reach a definitive diagnosis. In the literature, surgical resection is the preferred treatment for solitary PSH; however, for multiple PSHs, continuous follow-up and observation are crucial, as there is yet no effective treatment. Although rare, PSH should be considered when the suspected diagnosis contradicts the symptoms and results of auxiliary 
examinations. Therefore, physicians must remain highly vigilant in such cases to reach an accurate diagnosis and deliver effective treatment.

\section{Acknowledgements}

The authors would like to thank colleagues from the Imaging, Pathology, Laboratory and Thoracic Surgery Departments of General Hospital of Western Theater Command for help in diagnosing the patient.

\section{Funding}

No funding was received.

\section{Availability of data and materials}

All data generated or analyzed during this study are included in this published article.

\section{Authors' contributions}

HL was the patient's doctor. LM analyzed the data and wrote the article together with HL and TZ. YC was pathologist and confirmed the pathological diagnosis. DP, ZL and XD helped during the treatment and collected data. ZX and ZC directed the treatment of the patient, collection of data and writing of the article.

\section{Ethics approval and consent to participate}

All examinations and medical procedures were approved by the patient and her family.

\section{Patient consent for publication}

The patient agreed for the case study to be published.

\section{Competing interests}

The authors declare that they have no competing interests.

\section{References}

1. Liebow AA and Hubbell DS: Sclerosing hemangioma (histiocytoma, xanthoma) of the lung. Cancer 9: 53-75, 1956.

2. Ng WL and Ma L: Is sclerosing hemangioma of lung an alveolar mixed tumour? Pathology 15: 205-211, 1983.

3. Huszar M, Suster S, Herczeg E and Geiger B: Sclerosing hemangioma of the lung. Immunohistochemical demonstration of mesenchymal origin using antibodies to tissue-specific intermediate filaments. Cancer 58: 2422-2427, 1986.

4. Satoh Y, Tsuchiya E, Weng SY, Kitagawa T, Matsubara T, Nakagawa K, Kinoshita I and Sugano H: Pulmonary sclerosing hemangioma of the lung. A type II pneumocytoma by immunohistochemical and immunoelectron microscopic studies. Cancer 64: 1310-1317, 1989

5. Xu HM, Li WH, Hou N, Zhang SG, Li HF, Wang SQ, Yu ZY, Li ZJ, Zeng MY and Zhu GM: Neuroendocrine differentiation in 32 cases of so-called sclerosing hemangioma of the lung: Identified by immunohistochemical and ultrastructural study. Am J Surg Pathol 21: 1013-1022, 1997.

6. Katzenstein AL, Weise DL, Fulling K and Battifora H: So-called sclerosing hemangioma of the lung. Evidence for mesothelial origin. Am J Surg Pathol 7: 3-14, 1983.

7. Devouassoux-Shisheboran M, Hayashi T, Linnoila RI, Koss MN and Travis WD: A clinicopathologic study of 100 cases of pulmonary sclerosing hemangioma with immunohistochemical studies: TTF-1 is expressed in both round and surface cells, suggesting an origin from primitive respiratory epithelium. Am J Surg Pathol 24: 906-916, 2000.

8. Lee ST, Lee YC, Hsu CY and Lin CC: Bilateral multiple sclerosing hemangiomas of the lung. Chest 101: 572-573, 1992.

(i) $\Theta$ This work is licensed under a Creative Common Attribution-NonCommercial-NoDerivatives 4.0 International (CC BY-NC-ND 4.0) License. 